Economía, Sociedad y Territorio, vol. xx, núm. 63, 2020, 335-364.

DOI: https://doi.org/10.22136/est20201544

\title{
Trayectorias de habitabilidad en contextos de segregación socio-espacial: una aproximación teórico-metodológica desde las sensibilidades
}

\section{Trajectories of dwelling in contexts of socio- spatial segregation. A theoretical- methodological approach from sensibilities}

\author{
Ana Lucía Cervio*
}

\begin{abstract}
This paper discusses some theoretical connections between socio-spatial segregation, biographies and dwelling experiences identified in studies of sensibilities. Within a research project with residents of slums and informal settlements in the city of La Plata, Argentina, concepts of "experiences of dwelling" and "trajectories of dwelling" are defined, establishing some analytical correspondences for the observation of the current socio-spatial structuring. Then, a theoretical-methodological framework for studying "trajectories of dwelling" is proposed, reflecting on the limits and potentialities of the biographical method in the quest for urban segregation from individual experiences.
\end{abstract}

Keywords: biographies, experiences of dwelling, trajectories of dwelling, sensibilities, socio-spatial segregation.

\section{Resumen}

En el trabajo se exponen algunas conexiones teóricas entre segregación socioespacial, biografías y experiencias del habitar, identificadas desde los estudios de las sensibilidades. Con base en una investigación con residentes de villas de emergencia y asentamientos informales de la ciudad de La Plata, Argentina, se delimitan los conceptos experiencias del habitar y trayectorias de habitabilidad, utilizados en el estudio; se establecen correspondencias analíticas para la observación de la estructuración socio-espacial vigente; asimismo, se argumenta una propuesta teórico-metodológica para el abordaje de las trayectorias de habitabilidad, reflexionando sobre los límites y las potencialidades del método biográfico para la indagación de la segregación urbana desde las experiencias individuales.

Palabras clave: biografías, experiencias del habitar, trayectorias de habitabilidad, sensibilidades, segregación socio-espacial.

*Consejo Nacional de Investigaciones Científicas y Técnicas (Conicet)-Universidad de Buenos Aires (UBA), correo-e: anacervio@hotmail.com 


\section{Introducción ${ }^{1}$}

Junto con la gentrificación y la suburbanización de las metrópolis, la segregación socio-espacial es una preocupación creciente para los estudios sociales y las agendas públicas latinoamericanas. Pese a su emergente presencia, aún no existen consensos claros sobre su definición ni tampoco sobre opciones de medición que posibiliten alcanzar resultados consistentes, capaces de promover la construcción de evidencia empírica que permita análisis comparativos entre ciudades y países de la región. No obstante, las ostensibles "formas de desigual distribución de grupos de población en el territorio" (Lévy y Brun, 2002: 147) vuelven más que relevante recurrir (al menos preliminarmente) a una definición operativa que posibilite abordar la segregación socio-espacial como una de las manifestaciones de los procesos de reestructuración del capital en la actual fase de acumulación.

En el marco de esta inquietud, y atendiendo a las coordenadas definicionales ofrecidas más arriba, Sabatini et al. (2001: 27) definen la segregación como "el grado de proximidad espacial o de aglomeración territorial de las familias pertenecientes a un mismo grupo social, sea que éste se defina en términos étnicos, etarios, de preferencias religiosas o socioeconómicos, entre otras posibilidades". Anclando esta conceptualización en el marco del proceso más general de acumulación capitalista y sus metamorfosis a nivel territorial, puede afirmarse que la proximidad espacial que se sitúa en el "corazón" de la definición anterior no responde a un hecho estrictamente espacial (si por ello se entiende un espacio cosificado/reificado) sino que, por el contrario, debe ser comprendida como el resultado de procesos históricos de apropiación diferencial del suelo urbano por parte de las distintas clases y grupos sociales.

En esta línea, puede afirmarse que la segregación socio-espacial se manifiesta de dos maneras complementarias que producen consecuencias sociológicas particulares sobre el espacio de la ciudad y sobre las experiencias de sus habitantes: a) la homogeneidad social que revisten las subdivisiones socio-espaciales en las que queda "fracturada" la ciudad, y $b$ ), la concentración a escala urbana de los espacios residenciales ocupados por las distintas clases sociales. La articulación de ambas características acentúa las desigualdades socio-económicas -de las cuales la segregación es sólo una de sus manifestaciones-, promoviendo la configuración de zonas urbanas con un fuerte clivaje de clase (Cervio, 2015a).

\footnotetext{
${ }^{1}$ Este trabajo es una versión mejorada de una ponencia presentada en las X Jornadas Nacionales de Sociología de la Universidad Nacional de La Plata. Facultad de Humanidades y Ciencias de la Educación UNLP. Ensenada, 5, 6 y 7 de diciembre de 2018.
} 
En el marco reseñado, este trabajo se propone argumentar algunas conexiones entre segregación socio-espacial, biografías y experiencias del habitar. Para ello, se presenta una serie de viñetas teóricas y metodológicas elaboradas en el marco de una investigación en curso que busca reconstruir las trayectorias de habitabilidad y las sensibilidades a ellas asociadas. Trayectorias que portan residentes de villas de emergencia y asentamientos informales del Partido de La Plata, en sus tensiones con los procesos de estructuración socio-espacial vigentes. La estructura expositiva diseñada es la siguiente: en primer lugar, se define la noción de experiencia del habitar desde una perspectiva teórica centrada en el análisis de las políticas de las sensibilidades que organizan la vida en las ciudades; en segundo lugar, se presenta una aproximación teórica al estudio de las trayectorias de habitabilidad de sujetos que viven en la periferia pobre de La Plata, Argentina. Finalmente, se introducen algunos desafíos teóricometodológicos que sugiere la indagación de la segregación urbana desde las experiencias individuales.

\section{Experiencia del habitar: una conceptualización posible}

Desde Nietzsche a Heidegger, pasando por Lefebvre, Bachelard y Bourdieu, sólo por citar algunos de las referencias ineludibles, habitar supone la apropiación y permanencia en el espacio por parte de los sujetos, en conexión con un conjunto de sentidos, relaciones, objetos y disposiciones que se juegan en torno a ello.

A diferencia de otros vocablos alrededor de los cuales discurren diversas e incluso contradictorias definiciones, la Real Academia Española (RAE, 2019) define habitar mediante una única acepción: vivir, morar. Tan concreta, pero a la vez genérica designación, hace del habitar una experiencia fundante en torno de la cual transcurre la vida de los sujetos, sus logros, conflictos, avatares y deseos.

Recuperando sus orígenes etimológicos, habitar, no sólo se conecta con el acto de "ocupar un lugar" o "vivir en él” (lat. habitāre), sino también es frecuentativo del término haber (lat. habere). A partir del siglo XII, este verbo "capital” para la gramática espańola será progresivamente definido como la acción de tener o poseer (Corominas y Pascual, 1984). ${ }^{2}$ De modo que habitar es, además de ocupar un lugar, poseerlo, disponerlo, disputarlo, disfrutarlo.

\footnotetext{
${ }^{2}$ Junto con las definiciones asociadas a la morada y la posesión antes comentadas, habitar también forma parte de una familia de palabras que incluye al hábito (vestido o traje que usan religiosos y religiosas; insignia con que se distinguen las órdenes militares) y los hábitos (costumbres, destrezas).
} 
Siguiendo a Lefebvre, habitar es "convertir" el espacio vivido en un lugar, volviéndolo propio. Tal apropiación es un acto creativo transformador que se precipita sobre el espacio y sobre los sujetos, involucrando por ello un fuerte componente emocional, simbólico e imaginario.

De acuerdo con el sociólogo francés, el sentido moderno del habitar pudo ser recuperado gracias a la mediación de Nietzsche y Heidegger. Con sus teorizaciones, ambos filósofos contribuyeron a poner fin a la mirada reduccionista que durante el siglo XIX concebía al habitar humano como el (simple) "lugar de habitación” y restringía su complejidad inherente a un conjunto de actividades elementales: comer, dormir, reproducirse, etc.

Según Lefebvre, con Heidegger (1951) se rehabilita "el habitar" como base fundamental del ser. En efecto, para el citado pensador alemán, habitar es el fin último que persigue toda acción de construir. Sin embargo, envueltas en una sencilla y unidireccional relación de medio-fin, construir y habitar no constituyen acciones separadas, sino que se penetran mutuamente, provocando relaciones esenciales, al punto que: "construir no es sólo medio y camino para el habitar. El construir ya es, en sí mismo, habitar" (Heidegger, 1951: 1).

En esta línea de pensamiento, habitar es un "acontecer" que comienza con la construcción pero que la trasciende, a la vez que incluye/contiene/ expresa originariamente la manera según la cual los hombres son en la tierra, es decir, los modos cómo éstos experiencian lo habitual. En otros términos, para Heidegger habitar es un rasgo fundamental del ser; la condición más elemental por la cual el hombre accede a su dasein (ser-ahí). Habitar da cuenta de la totalidad existencial y, por lo tanto, es comprendido como un permanecer que concede humanidad, dejando que las cosas surjan en torno al ser.

Ahora bien, así como el habitar señala cómo se experiencia ese permanecer y residir "terrenal" asociado con el ser, para Heidegger también engloba una dimensión "poética" que conecta a los hombres con lo posible y lo imaginario.

Comentando el admirable poema de Hölderlin, "Poéticamente habita el hombre", Heidegger declara que la palabra del Poeta no se refiere en absoluto a las actuales condiciones de la habitación. No afirma que habitar quiera decir alojarse. Nos encontramos, dice Heidegger, ante una doble exigencia y un doble movimiento: pensar la existencia profunda del ser humano partiendo del habitar y de la habitación - pensar el ser de la Poesía como un "edificar", como un "hacer habitar" por excelencia (Lefebvre, 1978a: 152).

En otras palabras, habitar no es simplemente alojarse. No es una función accidental del hombre, sino una de sus manifestaciones esenciales y 
definitorias. Se trata de un rasgo antropológico ineludiblemente sujeto a los cambios en las relaciones y modos de producción. De manera que la historia del habitar puede ser definida como un capítulo (y no menor) de la historia social y económica de la humanidad (Lefebvre, 1978a).

De acuerdo con Lefebvre, habitar es una práctica creativa y productiva. Como tal, es producto-producción de un complejo proceso de apropiación espacial que se expresa "objetivamente" en un conjunto de productos, cosas, sentidos y locuciones. Dicha apropiación -que exige una producción, así como la necesidad y el "deseo de hacer"- designa a la totalidad de prácticas sociales que confieren a un determinado espacio las cualidades propias de un lugar, de una obra ${ }^{3}$ (Lefebvre, 2013). La apropiación aquí no se define desde la posesión sino desde un hacer/producir.

En este marco, habitar es apropiarse del espacio, convirtiéndolo en lugar. Es adaptar, usar, transformar y verter en el espacio la afectividad del usuario, la imaginación del habitante. De allí que, junto con los objetos, los sentidos y las palabras, en el habitar las emociones desempeńan un papel central.

Habitar, para el individuo o para el grupo es apropiarse de algo. Apropiarse no es tener en propiedad, sino hacer su obra, modelarla, formarla, poner el sello propio (...). Habitar es apropiarse un espacio; es también hacer frente a los constreñimientos, es decir, es el lugar del conflicto, a menudo agudo entre los constreńimientos y las fuerzas de apropiación (...) Cuando el constreńimiento impide cualquier apropiación, el conflicto desaparece o casi desaparece. Cuando la apropiación es más fuerte que el constreńimiento, el conflicto desaparece o tiende a desaparecer en un sentido. En otro sentido, estos casos de superación de los conflictos son casos límites y casi imposibles de alcanzar; el conflicto entre apropiación y constreñimiento es perpetuo (...) los interesados lo resuelven en otro plano, el de la imaginación, el de lo imaginado. Cualquier ciudad, cualquier aglomeración, ha tenido y tiene una realidad o una dimensión imaginaria (...)es necesario hacer un sitio a estos sueños, a este nivel de lo imaginario, de lo simbólico, espacio que tradicionalmente ocupaban los monumentos (Lefebvre, 1978a: 210).

A diferencia del hábitat, ${ }^{4}$ el habitar (se) configura (en) la relación de los sujetos con y a través de su espacio vivido y, como tal, se configura en su propio despliegue (siempre) creativo, cotidiano, múltiple y conflictivo.

Desde la perspectiva de Lefebvre (2013), el espacio vivido (aquel en el que los habitantes ponen en juego dimensiones simbólicas e imaginarias

\footnotetext{
${ }^{3}$ En la propuesta teórico-política lefebvriana, “obra” no significa objeto de arte, sino la actividad de un grupo que se apodera y se hace cargo de su papel y destino social; en definitiva, una autogestión creativa de la ciudad, de sus espacios, de sus ritmos, signos, símbolos y relaciones (Lefebvre, 1978b).

${ }^{4} \mathrm{Al}$ recuperar la visión heideggeriana, Lefebvre (1978a) sostiene que, considerándola en forma aislada, la función de alojamiento se acerca más al hábitat (el espacio construido, que se sitúa en el plano morfológico y normativo) que al habitar. Este último es comprendido como un rasgo antropológico fundamental asociado con lo creativo, lo lúdico y lo cotidiano. Así, mientras el hábitat es descripto como un "cuadro", el habitar es una "actividad", es decir, una situación vinculada con la apropiación.
} 
en sus búsquedas de nuevas posibilidades de la realidad espacial) se construye y tensiona con las estructuras sociales en las que éste se inscribe (como producto social), y las que al mismo tiempo son configuradas por él (como productor de lo social). En este marco, habitar es una experiencia en permanente re-definición, dependiente de los flujos y ritmos (sociales, políticos, económicos, culturales) que atraviesan y condicionan la vida cotidiana ${ }^{5}$ en las sociedades capitalistas. Sin embargo, esta condición "fluctuante" del habitar debe ser comprendida en el marco de los condicionantes estructurales (clase, etnia/raza, género, generación, etc.) que operan permanentemente en la estructuración de dicha experiencia histórica y geoculturalmente situada.

Conectando esta sintética referencia de la teoría lefebvriana del habitar con los aportes de una sociología de los cuerpos/emociones, es indudable que toda práctica social, entre ellas habitar, es desplegada por cuerpos/ emociones. ${ }^{6}$ Así, el proceso general de hacer la ciudad -en el que confluyen distintas dinámicas histórico-sociales que se articulan o contradicen con eje en el conflicto, el deseo, la necesidad y el disfrute de las y los usuarioshabitantes- es protagonizado por cuerpos percipientes, sintientes, hacientes (Lindon, 2009) que encarnan y actualizan los mecanismos y efectos de dominación vigentes. De allí el interés que persigue este trabajo de analizar los procesos corporales/emocionales involucrados en las experiencias del habitar que tienen lugar en escenarios urbanos capitalistas.

Desde la perspectiva aquí asumida, el juego entre impresiones y percepciones que provienen del intercambio con el ambiente, conforma particulares modos de ver, oír, gustar, tocar y oler que definen, condicionando, las emociones que la ciudad posibilita, o bien, coarta entre los sujetos que la habitan (Cervio, 2015b). Aunque en primera instancia se vivencien en forma individual, ya en el siglo XIX, Marx (2010) mostró cómo los sentidos corporales y las prácticas que ellos posibilitan o restringen son productos sociales, históricos y geo-culturales construidos por y desde la posición de clase de los sujetos.

Partiendo de la condición espacial, corporal y emocional de toda práctica, el cuerpo/emoción no sólo es constitutivo e inherente a la acción, sino también una forma de espacialidad que moldea (y es moldeada por) la matriz tiempo-espacio a partir de la cual el sujeto vivencia, narra y clasifica el mundo social en general, y las experiencias en/con la ciudad en particular (Lindon, 2009).

${ }^{5}$ La vida cotidiana es comprendida por el autor como la auténtica y genuina práctica de creación del espacio y de la vida social (Lefebvre, 1972b).

${ }^{6} \mathrm{El}$ análisis que aquí se presenta se inscribe en un posicionamiento teórico-epistémico que parte de reconocer la relación insoslayable que existe entre cuerpos y emociones para la comprensión de los procesos de estructuración social (Scribano, 2013). 
Así, las prácticas espaciales desplegadas por el cuerpo conllevan una dimensión del orden del sentir que conecta la construcción social de la ciudad y de sus espacios con las políticas de las sensibilidades que producen y sobre las que operan las tramas de la dominación capitalista. Las mencionadas políticas pueden ser comprendidas como "el conjunto de prácticas sociales cognitivo-afectivas tendientes a la producción, gestión y reproducción de horizontes de acción, disposición y cognición" (Scribano, 2017: 244). Así, como prácticas, las sensibilidades organizan la vida cotidiana y las maneras de ordenar las preferencias y los valores de los sujetos, a la vez que definen parámetros para la gestión del tiempo-espacio en el que se inscriben las interacciones sociales.

Desde este marco de entendimiento, este trabajo parte de un supuesto inicial: las sensibilidades se conectan con las experiencias del habitar que se producen y reproducen en las ciudades. Las mismas, no se circunscriben a la mera función de alojamiento, sino que designan y son resultado de las condiciones materiales y emocionales involucradas en el habitar como práctica social y de clase (Lefebvre 1978a, 2013).

De esta manera, puede afirmarse que las ciudades enuncian, encarnan y producen sociabilidades y vivencialidades múltiples. ${ }^{7}$ En sus tensiones, los aludidos procesos estructurantes de lo social configuran sensibilidades y experiencias diversas que inciden en la gramática de las acciones de los cuerpos que se cruzan, se conectan, se friccionan, se huelen, se tocan, se gustan, se miran, se oyen (o no). Así, emerge una variada gama de relaciones, objetos y conflictos que se espacializan para hacer de la ciudad un lugar sentido por y a través del cuerpo, en permanente coconstitución intersubjetiva.

Dado que el mundo se conoce por y a través del cuerpo, los ojos, los oídos, la nariz, la boca y la piel son locus que hacen posible el contacto entre el cuerpo y el mundo (Le Breton, 2009). Llevado al plano urbano, las ciudades pueden ser comprendidas como paisajes visuales, sonoros, olfativos, gustativos y táctiles que, analizados en su conjunto, permiten comprender la sensibilidad como una formación histórica, y la experiencia como un campo multisensorial socialmente definido.

Desde esta perspectiva, la experiencia del habitar es aquí comprendida como una

relación sensible -viabilizada por la acción y potencia de los sentidos- que alude a los entramados prácticos y emocionales que los sujetos ponen en juego en sus

7 "La sociabilidad es una manera de explicar los modos que al inter-actuar los agentes viven y con-viven. La vivencialidad es una manera de expresar los sentidos que adquiere el estar-en-cuerpo con otros como resultado, por un lado, del 'experienciar' la dialéctica entre cuerpo individuo, social y subjetivo; y, por otro lado, de las lógicas de apropiación de las energías corporales y sociales” (Scribano, 2010: 174) 
interacciones cotidianas. En términos generales, dicha experiencia es el resultado de la in-corporación de los procesos y efectos de dominación (vueltos mirada, olfacción, audición, tacto y gusto) que actualizan las percepciones asociadas a las formas socialmente construidas de las sensaciones. (Cervio, 2015b: 43)

De este modo, experienciar la ciudad y los espacios que se habitan, lejos de remitir a un acto particular/individual, señala los modos socialmente construidos y aceptados de gestionar la distribución y disposición de clase de los cinco sentidos que organizan la vida social en general, y la vida urbana en particular.

\section{Trayectorias de habitabilidad en contextos de socio-segregación}

Como se ha referido en la introducción, la segregación socio-espacial es una dimensión particular de los procesos de estructuración social que tienen lugar en las ciudades capitalistas (Sabatini et al., 2001). De ahí que el análisis de este fenómeno, más allá (o más acá) de los diversos indicadores estadísticos elaborados para dar cuenta del mismo, no puede prescindir del contexto socio-histórico en el que se produce, ni tampoco desconocer las complejas vinculaciones entre estructura espacial y estructura social que la segregación pone en evidencia.

En términos conceptuales en el marco de esta indagación, la segregación socio-espacial supone la confluencia de tres dinámicas concretas. En primer lugar, trasluce particulares mecanismos de diferenciación, pues: "la división social del espacio tiene como componente fundamental la característica de ser la expresión espacial de la estructura de clases o de la estratificación social" (Duhau, 2003: 179). De modo que, cuando se observan las particularidades que asume la distribución poblacional en ciertas áreas urbanas, o bien cuando se evalúan las magnitudes y cualidades que reviste la concentración de ciertos grupos en áreas específicas de la ciudad, la diferenciación aludida supone un concreto posicionamiento de los sujetos y de los espacios que obliga a complejizar el concepto y a considerar la segregación como criterio de jerarquización social.

Esto conduce a una segunda dinámica confluyente, en donde la segregación es una expresión espacial de la desigualdad social. Esta última es observable, por ejemplo, a partir de la coexistencia de áreas que limitan o restringen los desplazamientos y las circulaciones de ciertos grupos, lo que pone en evidencia un profundo proceso de zonificación territorial de acuerdo con parámetros socioeconómicos claramente definidos (Prévôt Schapira, 2002; Saraví, 2008). De forma que, además de diferenciación y desigualdad, la segregación provoca un radical desencuentro entre clases, es 
decir, la virtual cancelación de sociabilidades entre sujetos que se reconocen "otros-extraños". Tal espacialización de la alteridad radical promueve, en adición, la expansión de la desconfianza, el temor y el extrañamiento como sensaciones extendidas que refuerzan, desde el plano del sentir, los múltiples muros, bordes y fronteras que atraviesan y configuran a la "ciudad segregada" (Cervio, 2015a).

Analizadas desde la segregación como un vector estructural sustantivo, las ciudades capitalistas muestran las complejidades y los conflictos asociados con la diferenciación, la desigualdad y los desencuentros entre clases como dinámicas sociales confluyentes. Dinámicas que, además de organizar buena parte de las sociabilidades urbanas, se entrelazan en el ordenamiento espacial, incidiendo en la distribución diferencial del acceso a bienes y servicios urbanos, así como en el establecimiento de los sentidos que asumen las relaciones de proxemia y diastemia social (Cervio, 2019). Es precisamente para observar las complejidades mencionadas que en el marco de la investigación en curso se ha optado por estudiar las trayectorias de habitabilidad de mujeres y varones platenses que, desde hace varias generaciones, reproducen su cotidianeidad en contextos urbanos socio-segregados.

El Partido de La Plata, capital de la provincia de Buenos Aires, es una ciudad intermedia. Situada a 60 kilómetros de la Ciudad Autónoma de Buenos Aires, posee -según el último censo nacional- una población total de 654,324 habitantes, distribuidos en sus diversas localidades (INDEC, 2012).

Desde hace al menos tres décadas, en La Plata se verifica un sistemático proceso de urbanización informal, encabezado por quienes no tienen acceso a la compra de una vivienda en el mercado formal ni tampoco son beneficiarios de políticas habitacionales estatales. Así, los elevados precios del suelo en el mercado inmobiliario, junto con la desocupación y la informalidad laboral que dominan como signo las condiciones socioocupacionales de los sectores populares, explicarían, en gran medida, el surgimiento de urbanizaciones espontáneas ${ }^{8}$ y el dominio de la informalidad como condición residencial en la periferia pobre de La Plata.

\footnotetext{
${ }^{8}$ Si bien la "espontaneidad" es uno de los rasgos que asume gran parte de las urbanizaciones informales localizadas en la periferia platense, llegando a ser, incluso, una adjetivación ampliamente utilizada por investigaciones locales (Rodríguez, 2018; Frediani, 2013), aquí se sostiene la necesidad de revisar críticamente las complejidades inherentes a dicho rasgo, explorando las particularidades de cada caso, desde la perspectiva de los sujetos. En efecto, el estudio de los asentamientos informales y de las historias de vida que allí surgen impone examinar las articulaciones entre estructura espacial, estructura social y trayectorias individuales, revisando una serie de interrogantes referidos al periodo de surgimiento, las motivaciones de sus habitantes, la tipología de los terrenos, la modalidad de ocupación, las características infraestructurales, las políticas urbanas vigentes, etc. En suma, dada la centralidad que revisten para esta investigación las conexiones entre procesos estructurales y biogra-
} 
Reconociendo los matices y las diferencias que exhibe la historia del hábitat precario en los interiores provinciales, en términos generales, puede afirmarse que en Argentina se observan dos grandes tipologías de urbanización informal: las villas de emergencia y los asentamientos. Las primeras son ocupaciones irregulares de tierra urbana vacante que se caracterizan por poseer una alta densidad poblacional. En general, presentan una trama irregular con acceso a las viviendas a través de pasillos, y el proceso de ocupación suele ser individual y diferido en el tiempo por parte de pobladores que conciben a la villa como una solución habitacional transitoria. Por su parte, los asentamientos informales implican la producción de lotes mediante la ocupación de tierras vacantes, en su mayoría privadas. El trazado suele ser regular y planificado. Dado que la ocupación de la tierra exige vivir allí, "las viviendas presentan una evolución desde simples 'taperas' a construcciones firmes, dependiendo sus características de la capacidad y recursos de quienes la habitan" (Varela y Cravino, 2008: 60). Las familias auto-producen de diversas maneras las infraestructuras y viviendas, aunque observando las normas vigentes. Por lo general, existe perspectiva de regularización a través de la intervención del Estado, y el proceso de ocupación suele ser colectivo (Rebord, 2006).

En términos cualitativos y cuantitativos, la creciente informalidad urbana a la que se viene asistiendo en Argentina durante las últimas décadas, evidencia un profundo proceso de fragmentación, ${ }^{9}$ y segregación social al interior de las ciudades. Datos elaborados por la Organización TECHO (2016), a partir de un relevamiento en 11 territorios -que representan el $67 \%$ de la población total-, muestran que a nivel país se registran 2432 asentamientos informales. Tomando como base la media nacional de 4.6 miembros por familia, el informe estima que esos aglomerados están habitados por 2,993,151 personas, es decir, unas 650,685 familias. De ese total, $73 \%$ de las familias no cuenta con acceso formal a

fías, reconstruir las dinámicas sociales, políticas, económicas y culturales que han confluido para la formación de los asentamientos en estudio es un objetivo ineludible.

${ }^{9}$ La noción de fragmentación surge para dar cuenta de los efectos de la globalización sobre la estructura urbana. Como tal, señala la aparición de nuevas centralidades que se imponen como característica tanto en las metrópolis como en las ciudades intermedias, multiplicando ("haciendo estallar") el funcionamiento integral/total de la ciudad en múltiples y diferenciadas unidades (Prévôt Shapira, 2001). En el caso latinoamericano, desde la década de los 80 se está observando de manera progresiva la aparición de ciudades multipolares en las que las nuevas centralidades no sólo son "aprovechadas" con fines residenciales (patrón común de los procesos de sprawlo suburbanización), sino que, fundamentalmente, emergen como nuevos centros destinados a la producción, consumo, recreación, etc. (Vecslir y Ciccolella, 2012). Este proceso de fragmentación ha supuesto la emergencia de "ciudades fragmentadas" en las que una organización más dispersa y menos jerárquica del espacio urbano da cuenta de dos dinámicas simultáneas. Por un lado, procesos macro asociados con la multiplicación y diversificación de los flujos y mercados mundializados y, por el otro, las contradicciones y disparidades socio-espaciales que surgen como producto de las nuevas formas de crecimiento urbano (Borsdorf e Hidalgo, 2010). 
la red de energía eléctrica, 98\% no accede a la red cloacal y 95\% no tiene acceso al agua corriente.

En 2015, la Subsecretaría Social de Tierras, Urbanismo y Vivienda, dependiente del Ministerio de Infraestructura y Servicios Públicos de la Provincia de Buenos Aires, elabora el Registro Público de Villas y Asentamientos de la Provincia de Buenos Aires (Gobierno de la Provincia de Buenos Aires, 2015). ${ }^{10}$ De acuerdo con este relevamiento, en la provincia se identifican 1805 barrios calificados como "informales", ${ }^{11}$ habitados por unas 423,836 familias. En particular, el Partido de La Plata es el primer distrito provincial en cantidad de urbanizaciones informales y el segundo en cantidad de habitantes residiendo en dichas urbanizaciones: ${ }^{12}$ se registran 167 (33 villas, 108 asentamientos y 26 correspondientes a otras tipologías) en las que habitan 21,616 familias que reproducen su cotidianeidad en el marco de una profunda informalidad e inseguridad urbana. ${ }^{13}$

En términos de la localización y distribución de las urbanizaciones informales, los datos recolectados por el informe provincial muestran que mayoritariamente se asientan en la zona sur del Partido, especialmente en las localidades de Melchor Romero, Villa Elvira, Los Hornos y Altos de San Lorenzo (figura 1).

La ocupación del suelo en los ejes SE y SO se explicaría, en primera instancia, por el hecho de que son los espacios urbanos más relegados en términos económicos, ambientales y de infraestructura de servicios urbanos del Partido; situación que se traduce en precios del suelo más bajos (Del Río, 2016). Además, debe destacarse que las urbanizaciones informales que se asientan en estos ejes se ubican relativamente próximas al casco urbano: "Esto tiene que ver con las posibilidades de accesibilidad al transporte y los servicios que ofrecen las vías principales que conectan con el casco urbano" (Rodríguez, 2018: 125). En suma, el corredor Sur platense ostenta la desigualdad en la distribución de recursos respecto al casco urbano y al eje Norte, los cuales no sólo presentan los valores más altos en relación a los precios del suelo, sino que, además, están estrechamente vinculados con el Área Metropolitana de Buenos Aires (AMBA) y la Ciudad Autónoma de Buenos Aires (CABA), a través de la Autopista Buenos Aires-La Plata.

\footnotetext{
${ }^{10}$ Cabe destacar que dicho Informe fue producido a instancias de la Ley Provincial de Acceso Justo al Hábitat (14.449), sancionada en 2012.

${ }^{11}$ Se detectaron 967 asentamientos precarios (en vías de regularización), 445 villas y 393 aglomeraciones tipificadas como "otros".

${ }^{12}$ La distribución la encabeza el partido de La Matanza, con 53,339 familias ocupando villas, asentamientos y otras tipologías.

${ }^{13}$ Según el informe de TECHO (2016), La Plata, segundo distrito más poblado de la provincia de Buenos Aires, es el que encabeza la distribución del número de urbanizaciones informales con 157.
} 


\section{Figura 1}

\section{Localización de urbanizaciones informales en el Partido de La Plata}

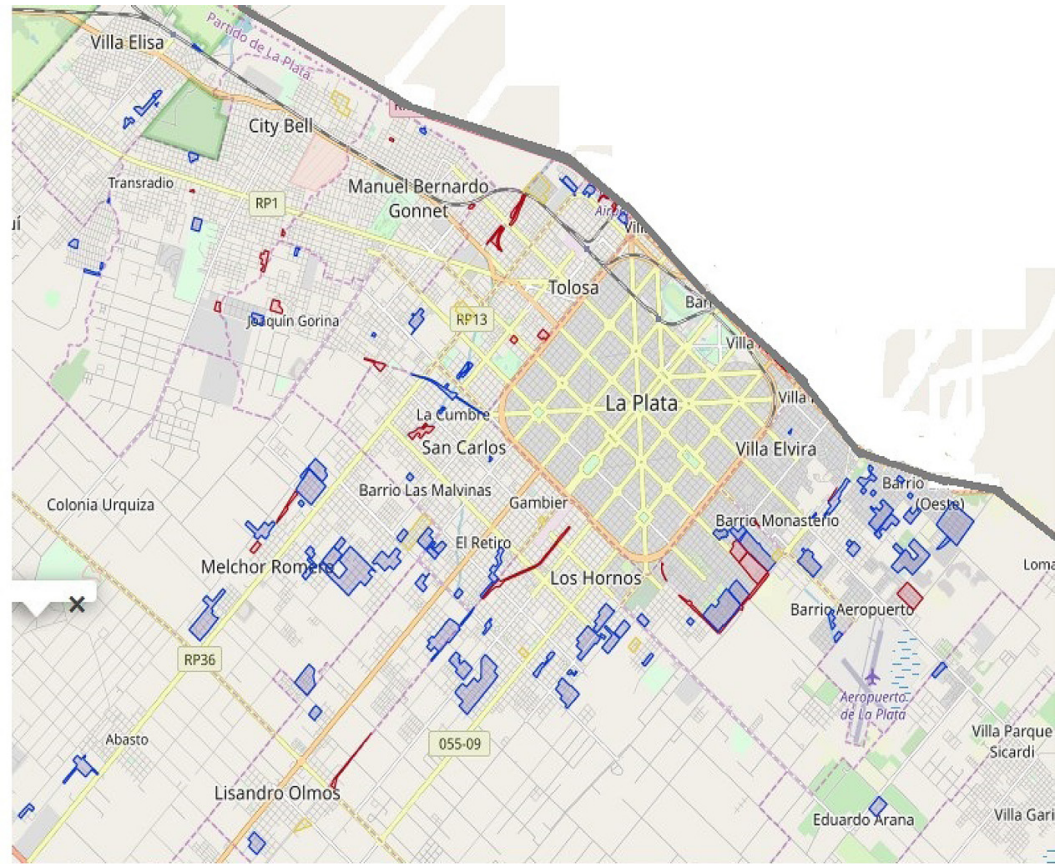

Fuente: Registro Público de Villas y Asentamientos de la Provincia de Buenos Aires, 2015.

Los datos anteriores actualizan las dinámicas asumidas por el crecimiento urbano en el Partido de La Plata durante los últimos 40 años, las cuales pueden sintetizarse grosso modo de la siguiente manera:

a) Expansión de la periferia a partir de un proceso de "desborde" poblacional desde el casco urbano. Esta expansión periférica por la vía de la ocupación de nuevas superficies de suelo produjo transformaciones en el tejido urbano emergiendo nuevas actividades económicas, sociales y culturales en áreas anteriormente rurales o semi-rurales. Así, grandes superficies destinadas a explotaciones primarias intensivas comenzaron a ser erigidas como destinos residenciales de la mano de intensos procesos de revalorización del suelo que propiciaron una acelerada especulación inmobiliaria (Frediani, 2010).

b) Progresiva ocupación diferencial del área periférica de acuerdo a posiciones y condiciones de clase. La expansión sub-urbana que se produce desde fines de la década de los ochenta no sólo reduce la superficie de tierras productivas, sino que a partir de su avance alienta la 
configuración de enclaves urbanos (cada vez más) desiguales. De esta manera, los procesos de revalorización de áreas estratégicas a partir de la obra pública, junto con la especulación inmobiliaria y la escasa regulación estatal en materia del suelo, en los últimos años han favorecido una sostenida variación en los precios de las tierras entre las diversas áreas del Partido, observándose una intensificación de la desigualdad entre el corredor Norte y Sur. Tal movimiento socioeconómico ha profundizado el escenario de segregación socioespacial en la región, identificándose en la periferia platense territorios diferenciales que consolidan formas de vida antitéticas: por un lado, la segregación auto-inducida de los sectores de más altos ingresos y, por el otro, la segregación estructural (por expulsión) de los pobres urbanos (Prévôt Schapira, 2002; Saraví, 2008; Frediani, Rodríguez Tarducci y Cortizo, 2018).

Desde los años ochenta -aunque intensificándose en los noventa y en las primeras dos décadas del siglo XXI-, las urbanizaciones informales fueron instalándose progresivamente como estrategias de ocupación del espacio urbano periférico platense..$^{14} \mathrm{La}$ innegable desigualdad en la distribución de recursos y servicios urbanos que revela la actual existencia de 167 asentamientos informales en el Partido pone en evidencia la configuración de ciudadanías múltiples, claramente definidas desde el goce y el disfrute diferencial (clasista) de las accesibilidades urbanas.

Tales enclaves de pobreza refractan la presencia sostenida durante las últimas (casi) cuatro décadas de "habitantes de segunda". Es decir, sujetos que, sin agua potable, sin luz, sin salud, ni educación ni trabajo, se encuentran materialmente postergados en sus capacidades de agencia y, por lo tanto, disponibles y en condiciones de disponibilidad (energética y social) para otros. Frente a la persistencia de la pobreza como rasgo estructurante de la ciudad y de sus espacios de vida, y también como disposición de minusvalía social que confisca la autonomía de los sujetos, preguntarse por las sensibilidades y experiencias que configura dicha "habitabilidad de segunda" resulta central.

Atendiendo a este escenario contextual y al interés de problematizar las conexiones entre sensibilidades y experiencias del habitar en espacios socio-

\footnotetext{
${ }^{14}$ Según datos ofrecidos por el Registro Público de Villas y Asentamientos de la Provincia de Buenos Aires (Gobierno de la Provincia de Buenos Aires, 2015), 38.5\% de las urbanizaciones informales del Partido de La Plata se conformaron entre el 2000-2010 y el mismo porcentaje lo hizo durante la década de 1990. En los años ochenta se conformó $8.4 \%$ y desde el 2011 en adelante se formó $13.2 \%$ de las villas y asentamientos que existen en la actualidad. El estudio también indica que en 1974 aparecieron dos asentamientos precarios en las localidades de City Bell (El Country) y Villa Elisa (YPF).
} 
segregados, se elaboró un proyecto de investigación, actualmente en curso; ${ }^{15}$ el cual se propone reconstruir y analizar las trayectorias de habitabilidad y las sensibilidades a ellas asociadas que portan residentes de villas de emergencia y asentamientos informales de la ciudad de La Plata, en sus tensiones con los procesos de estructuración socio-espacial vigentes.

En el próximo apartado se presenta una aproximación teórico-metodológica posible para el estudio de las trayectorias de habitabilidad, desde una perspectiva de análisis que enfatiza las conexiones entre experiencias del habitar y sensibilidades.

\section{Trayectorias de habitabilidad: una definición teórico- metodológica}

Las relaciones entre espacio y sociedad han sido una preocupación constante para las Ciencias Sociales. En particular, la temática de la movilidad espacial pone en evidencia las conexiones entre espacio y estructuración social (Bericat Alastuey, 1994), resultando ineludible una lectura rigurosa de las diversas tramas explicativas que participan en las trayectorias de los cambios socio-espaciales observados en una sociedad, o en un grupo en particular, en un periodo de mediana o larga duración.

La movilidad espacial es un rasgo de los sujetos y colectivos sociales. Entendida en primera instancia como una práctica de desplazamiento que se refracta sobre la dinámica urbana, reconfigurándola en múltiples sentidos, la movilidad alude a recorridos, más o menos habituales y sostenidos, emprendidos en forma individual o colectiva por los sujetos, así como a desplazamientos que se concretan como consecuencia de decisiones residenciales, económicas, afectivas y familiares que toman los sujetos en el decurso de sus bio-grafías.

En el primer sentido aludido, la movilidad espacial de las distintas clases sociales es un hecho sociológico que acontece a diario en todas las ciudades del mundo (Cervio y Vergara, 2017). Millones de recorridos particulares y colectivos entretejen los tiempos-espacios de las ciudades, demandando infraestructura y servicios (transporte, corredores, semaforización, controles viales, sendas peatonales, etc.); organizando prácticas ligadas al viaje (cálculos de los tiempos que implica "salir", "viajar" y "llegar"; previsión y disposición de dinero y otros recursos indispensables para desplazarse; autocuidados corporales y estrategias de seguridad, etc.), y también expresando conflictos asociados con la disputa por el espacio

\footnotetext{
${ }^{15}$ Cervio, Ana Lucía: "Sensibilidades y trayectorias de habitabilidad en villas y asentamientos informales de la Ciudad de La Plata”, Proyecto CIC- CONICET [2018-2020].
} 
público y por el acceso a bienes y servicios urbanos (frecuencias y estado de las unidades del transporte, condiciones de las vías de circulación, disponibilidad de caminos y conservación de sendas peatonales, etc.). La localización diferencial de los lugares de residencia y los espacios de reproducción cotidiana, como el trabajo o la educación, explican, en mayor medida, la movilidad como una de las principales características de las ciudades modernas (Sennet, 2007; Mongin, 2006). La dinámica que adquiere la distribución y configuración de las vías de movilidad "disponibles" condiciona los desplazamientos posibles, según la posición y condición de clase de los sujetos, emergiendo como uno de los conflictos urbanos más importantes. Esta lógica conflictual, vinculada con la movilidad, se manifiesta estructuralmente en la distribución diferencial de las posibilidades de acceso a medios y vías de transporte. Desde allí, tal conflictividad se extiende a la organización de la vida, en clave de los tiempos de los sujetos, es decir, en la cantidad de horas-vida que demandan los desplazamientos necesarios para la reproducción material de la existencia cotidiana en las ciudades. Y esto es, indudablemente, una cuestión de clase.

Ahora bien, junto con las estrategias de movilidad que los habitantes de las ciudades emprenden a diario para satisfacer sus más diversas necesidades, la teoría social también se ha encargado de describir e interpretar las dinámicas de movilidad residencial intraurbana, es decir, "aquellas prácticas espaciales que involucran cambios en el lugar de residencia en la ciudad" (Di Virgilio, 2009: 234). Cuando dichos desplazamientos son observados articulando su dimensión espacial con la temporalidad en la que se inscriben, el concepto de trayectoria residencial resulta un potente descriptor del conjunto de cambios que, en tal sentido, realizan los hogares o las personas a lo largo de su vida.

De acuerdo con Delaunay y Dureau (2004), todos los desplazamientos que incluye una trayectoria afectan, en mayor o menor medida, la tipología de la vivienda ocupada, su localización en el espacio de la ciudad, la situación de tenencia y la autonomía residencial de los sujetos. Cualquier variación registrada en uno o en todos los componentes citados, provoca alteraciones en la movilidad, dando origen a un nuevo trayecto residencial. En tal sentido, un hogar puede vivenciar modificaciones en la tenencia, sin por ello alterar la localización ni el tipo de residencia (es el caso, por ejemplo, de trayectorias promocionales en las que una familia pasa de ser inquilina a propietaria de la vivienda que ocupa).

Como toda trayectoria que los sujetos construyen a lo largo de su desempeño biográfico en las distintas zonas de la vida social en las que participan, la trayectoria residencial alude a una serie sucesiva de posiciones que, lejos de encadenarse de manera casual/azarosa, responde a un orden inteligible. Dicho ordenamiento está estrechamente vinculado con 
las dinámicas socio-estructurales y afectivas que atraviesan y ordenan el calendario biográfico de los sujetos: etapas del ciclo de vida, carrera profesional, historia familiar, ciclos económicos, entre otras.

Recuperando los aportes señeros de Bourdieu (1990), puede afirmarse que el orden que reciben los distintos trayectos residenciales por los que atraviesan los sujetos desde su nacimiento está ligado al propio devenir de sus bio-grafías, pues cada una de ellas "habla" de la historia social vuelta historia personal. Desde el posicionamiento teórico aquí asumido, esto implica considerar, en primer plano, las trayectorias de los sujetos en términos de su posición y condición de clase. Tal afirmación se desprende del supuesto de que -junto con el género, la generación y la raza- la clase social explica en gran medida los diversos cambios, desplazamientos, aventuras y avatares que acontecen en una vida, sean éstos residenciales, laborales, políticos, educativos o vinculares/afectivos.

En conexión con la noción de experiencia del habitar, definida en el primer apartado, el proyecto de investigación en curso se propone reconstruir y analizar las trayectorias de habitabilidad y las sensibilidades a ellas asociadas que portan residentes de villas de emergencia y asentamientos informales del Partido de La Plata, en sus tensiones con los procesos de estructuración socio-espacial vigentes. En diálogo con las contribuciones efectuadas por los estudios de "movilidad espacial" -especialmente desde la geografía, la antropología y la sociología urbana- la investigación opta por reconstruir las mencionadas trayectorias desde una sociología de los cuerpos/emociones.

Desde una perspectiva teórica, las trayectorias de habitabilidad aluden al conjunto de "soportes" (Martuccelli, 2007) materiales, vincularesafectivos y sensibles que caracterizan la vida cotidiana en los territorios, en los barrios y en los hogares, y que en su conjunto configuran las experiencias del habitar de los sujetos en clave biográfica. Tales trayectorias incluyen la movilidad residencial, ${ }^{16}$ pero ésta no las define en forma suficiente. Concretamente, no sólo interesa reconstruir y describir los recorridos habitacionales (intra e interurbanos) experimentados por los sujetos a lo largo de su vida, sino que también se busca detectar y caracterizar los "hitos de habitabilidad" que los sujetos identifican en sus propias líneas biográficas, los cuales exceden ampliamente los desplazamientos residenciales per se.

Los mencionados "hitos" -seleccionados, recordados, reconstruidos y narrados por los sujetos- señalan la presencia de "pruebas" (Martuccelli, 2007) o "puntos de inflexión" (Sautu, 1999) en el calendario personal,

${ }^{16}$ En el sentido de los aportes de Delaunay y Dureau (2004), comentados más arriba: tipología de vivienda, tenencia, localización y autonomía residencial. 
imponiendo algún tipo de cambio en el cursus de vida en relación con el trayecto pasado y, probablemente, con el futuro. Pueden ser desde una mudanza o la migración a una nueva ciudad, hasta un cambio de trabajo, pasando por la adquisición de una vivienda social, la participación en alguna organización comunitaria, la separación de la pareja o el nacimiento de los hijos. En suma, se trata de momentos "bio-gráficos", que se graban/marcan en las grafías corporales, suscitando emociones diversas que irrumpen en la linealidad de la vida y suponen algún tipo de modificación/alteración en las formas rutinarias del habitar, esto es, en los modos hasta entonces conocidos de apropiación y creación del espacio que involucran en forma radical diversos registros de lo material, lo sensible y lo emocional (Lefebvre, 2013; Cervio, 2015b).

\section{Cuadro 1 \\ Experiencias del habitar, trayectorias e hitos de habitabilidad}

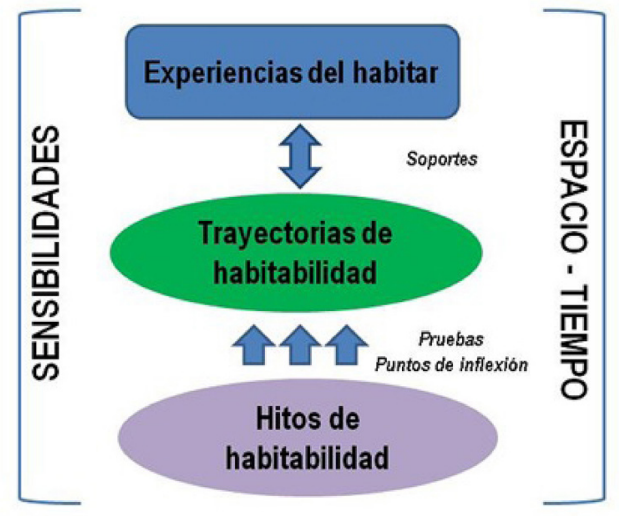

Fuente: Elaboración propia con base en el análisis precedente.

En referencia a la creciente singularización de las trayectorias personales que la sociedad actual impone sobre diversos dominios (consumo, producción, sociabilidades, etc.), Danilo Martuccelli (2007) sostiene que la sociología del siglo XXI debe (re)posicionar su mirada sobre los fenómenos sociales, otorgando centralidad a los horizontes analíticos que se abren desde las experiencias individuales. En esta línea, entre las diversas gramáticas del individuo, el autor privilegia el estudio de la individuación, es decir, el análisis de los procesos histórico-sociales que se articulan/ confluyen/tensionan para la constitución del individuo en un periodo histórico dado. 
La dinámica esencial de la individuación combina un eje diacrónico con un eje sincrónico, tratando de interpretar en el horizonte de una vida -o de una generación- las consecuencias de las grandes transformaciones históricas. La explicitación entre estos dos ejes explicita la personalidad de esta perspectiva, a saber, la interrogación por el tipo de individuo que fabrica estructuralmente una sociedad (Martuccelli, 2007: 30).

En su conformación como individuos, los sujetos enfrentan un conjunto de "pruebas" existenciales o "desafíos comunes", pero desde posiciones diversas y transitando experiencias disímiles (Araujo y Martuccelli, 2012). Se trata de retos históricos, socialmente producidos y desigualmente distribuidos, que los sujetos se ven compelidos a afrontar/resolver desde su singularidad, en aras de su "fabricación" como individuos (por ejemplo, escuela, trabajo, familia, ciudad). De este modo, la noción de "prueba" permite abordar la articulación entre procesos sociales y experiencias individuales, es decir, entre la estandarización estructural y la singularización de las experiencias.

\begin{abstract}
El estudio de la individuación por las pruebas no se reduce pues jamás a un mero estudio de trayectorias de vida y a una sucesión biográfica de etapas (formación, trabajo, jubilación). Apoyándose en él se propone, por el contrario, una macrosociología a escala de los individuos: estudiar una sociedad consiste en conocer el conjunto estandarizado de pruebas que le es específico (Martuccelli y de Singly, 2012: 76).
\end{abstract}

En esta línea, el análisis de las pruebas sociales posibilita la articulación en dos niveles: por un lado, permite identificar y analizar los principales desafíos que imperan en una sociedad en un periodo dado, partiendo de la experiencia de los individuos; por otro, posibilita estudiar las formas en que los individuos lidian con las pruebas, al ensayar respuestas diferenciales y movilizando distintos tipos de recursos para intentar resolverlas.

Para comprender los procesos de individuación, junto con las pruebas, Martuccelli plantea la importancia capital que detentan los "soportes". Éstos son definidos como los medios afectivos, materiales y simbólicos mediante los cuales el individuo llega a "sostenerse" en el mundo, es decir, el conjunto de elementos legítimos/ilegítimos, visibles/invisibles, confesables/inconfesables que conectan al actor con la vida social: el trabajo, la pareja, los hijos, la ideología, la participación social, los consumos y la profesión son algunos ejemplos en este sentido. La selección y operación de estos soportes en el seno de una vida posibilita -al decir del autor- la construcción de una "ecología existencial" singular que debe ser "leída" en clave de la gramática de individuación. 
Lo importante es la manera cómo los individuos se constituyen un entorno existencial combinando relaciones u objetos, experiencias o actividades diversas, próximas o lejanas, que, en la ecología así constituida, van o no a dotarse de significaciones absolutamente singulares. Este entramado heterogéneo y proteiforme crea alrededor de cada uno de nosotros un tejido existencial y social elástico que es, en el sentido a la vez más estricto y restringido del término, "nuestro" verdadero mundo (Martuccelli, 2007: 81).

En síntesis, las "pruebas" y los "soportes" son operadores analíticos que posibilitan interpretar las maneras en que lo social se estructura a nivel de las trayectorias y experiencias individuales. Mientras las primeras constituyen los desafíos estructurales a través de los cuales los individuos son producidos y se producen, los segundos son definidos como los medios a partir de los cuales el individuo llega a "sostenerse" en el mundo.

Con base en los aportes de Martuccelli sintetizados, en el marco de la investigación en curso se sostiene que los acontecimientos biográficos son analizadores válidos de los procesos de estructuración social, de las pruebas existenciales que la sociedad impone a sus individuos en un periodo dado, y de los soportes que éstos movilizan para "sos-tenerse" en la vida social. Dado el actual escenario social, signado por una creciente singularización de las experiencias, el estudio de las trayectorias biográficas se torna un interesante camino heurístico, pues posibilita captar los procesos estructurales a escala del individuo. Esto no implica ni individualismo metodológico ni tampoco matizar el clivaje de clase que atraviesa las experiencias. Lo que se busca es construir una estrategia de indagación que permita aprehender sociológicamente los distintos "nodos" experienciales que, en su articulación bio-gráfica, configuran las trayectorias de habitabilidad de los sujetos y las sensibilidades a ellas asociadas.

Atendiendo a las dimensiones biográficas y sensibles que interesan indagar, y reconociendo que las trayectorias de vida y habitacionales no son ni pueden ser "escindibles" del contexto socio-histórico en el que se despliegan (Wright Mills, 2003), la investigación sigue una estrategia metodológica cualitativa, basada en la realización de relatos biográficos a través de entrevistas en profundidad. ${ }^{17}$ Dicha estrategia de recolección de datos busca potenciar interpretaciones que den cuenta sociológicamente

${ }^{17}$ Se planifica la realización de entrevistas a varones y mujeres residentes en diversas villas y asentamientos informales de La Plata, con base en un muestreo cualitativo intencional de máxima heterogeneidad. El número de relatos a realizar quedará sujeto al criterio de saturación teórica. Por su parte, se prevé la concreción de al menos cuatro encuentros sucesivos con cada uno de los informantes, con el objetivo de elaborar los relatos biográficos y someterlos a la consideración de sus protagonistas. Esta actividad supone transcribir las entrevistas luego de cada encuentro y compartir el texto obtenido con los sujetos, a fin de proceder a realizar los ajustes que los mismos consideren convenientes, de acuerdo con sus propias apreciaciones/opiniones sobre las experiencias vitales narradas en cada una de las entrevistas. Los resultados de estas revisiones parciales serán los insumos a utilizar para la elaboración final de cada relato biográfico. 
de las maneras en que distintos procesos sociales permean y configuran experiencias vivenciadas y narradas por los sujetos como "íntimas", "subjetivas" y "personales".

Como se anticipó, el estudio de la individuación requiere dar cuenta de la inscripción de las transformaciones estructurales en las trayectorias individuales, de aquí que el enfoque biográfico emerja como una opción epistémica y metodológica adecuada. En particular, los relatos biográficos promueven el despliegue narrativo de las experiencias vividas por los sujetos a lo largo del tiempo, constituyéndose, por ello, en un instrumento válido para el estudio de las sensibilidades.

A través de entrevistas sucesivas, esta técnica de investigación no persigue la recolección anecdótica de experiencias recordadas/evocadas/significadas por los sujetos, sino más bien procura elaborar un relato en primera persona que reconstruya los acontecimientos significativos dentro del "calendario vital" de los sujetos, en sus conexiones con los procesos de estructuración social (Sautu, 1999; Pujadas Muñoz, 1992). De manera que la elección de esta técnica se fundamenta en sus potencialidades epistémicas y metodológicas para elaborar interpretaciones sobre las experiencias del habitar que portan los sujetos en un periodo de mediana duración, enfatizando una lectura sociológica de los fenómenos sociales por y desde las experiencias individuales.

\section{Conclusiones}

En el marco del proceso de segregación socio-espacial que se ha venido profundizando durante las últimas décadas en La Plata -avalado, entre otros indicadores, por la multiplicación y densificación poblacional de villas y asentamientos informales localizados, preferentemente, en el Eje Sur del Partido- este trabajo se propuso interpelar, desde una mirada sociológica, las vinculaciones entre segregación socio-espacial, biografías y experiencias del habitar emergentes en el contexto de una investigación actualmente en desarrollo. En tal sentido, lejos de ofrecer el análisis de resultados finales, el objetivo general fue dar cuenta de una propuesta teórico-metodológica para el abordaje de las trayectorias de habitabilidad elaborada desde el campo de los estudios sociales de las sensibilidades.

La revisión de datos secundarios efectuada posibilitó elaborar un diagnóstico sobre el crecimiento urbano del Partido de La Plata durante los últimos 40 años, concluyéndose que el mismo es el resultado de un doble proceso de larga duración: a) la expansión periférica a partir del "desborde" poblacional desde el casco urbano, y b) la progresiva ocupación diferencial del área periférica de acuerdo a posiciones y condiciones de clase. 
Frente a tal diagnóstico, se sostuvo que la indagación dinámica de los mencionados procesos posibilita observar la confrontación de las historias individuales con la propia historia de la ciudad: una ciudad en la que la segregación y la fragmentación socio-espacial se combinan con la ocupación del espacio que realizan las distintas clases sociales y con la expansión/ crecimiento urbano que se "desborda" hacia la periferia, obstaculizando el acceso a una variada gama de derechos y consumos por parte de los grupos más pobres.

En este marco, el proyecto de investigación en curso se pregunta por las maneras en que dichos fenómenos estructurales son y han sido vivenciados por los sujetos en el transcurso de sus vidas. La apuesta es establecer enlaces analíticos entre la estandarización estructural y la singularización experiencial, identificando los modos en que la segregación socio-espacial atraviesa, configura y condiciona en forma dinámica las experiencias y sensibilidades de mujeres y varones que habitan desde hace años en la periferia pobre platense. ${ }^{18}$ En suma, el desafío es reponer, desde la mirada de los sujetos, los modos en que la segregación socio-espacial fue materializándose en trayectorias de habitabilidad (a primera vista, únicas, singulares e irrepetibles).

Desde la opción teórica suscripta, comprender las trayectorias de habitabilidad exige identificar, reconstruir y describir los "soportes" materiales, vinculares-afectivos y sensibles que los sujetos han puesto en juego en sus experiencias del habitar a lo largo de sus vidas.

Como se definió al comienzo de este trabajo, las experiencias del habitar son entendidas como una relación sensible -viabilizada por la acción y potencia de los cinco sentidos- que alude a los entramados prácticos y emocionales que los sujetos ponen en juego en sus interacciones cotidianas. Dichas experiencias son resultantes de la in-corporación

\footnotetext{
${ }^{18}$ Esta pregunta remite, inexorablemente, a una serie de articulaciones teóricas que, por razones de espacio, aquí sólo se enuncian, pero que serán abordadas en la investigación a partir de las observaciones empíricas. En el contexto actual de un capitalismo que se expande a merced de profundas y renovadas estrategias de expulsión, desposesión y expropiación de energías vitales y sociales (algunas son explícitas, como ilustran millones de migrantes, desplazados, trabajadores esclavizados y segregados urbanos; otras, en cambio, se despliegan silenciosas y desapercibidas en la cotidianeidad de la vida), ¿cuáles son las características que asumen los procesos de individuación en el Sur Global, y cuáles son los límites y potencialidades que reviste el método biográfico para poder "captarlas" en su complejidad? La aludida dinámica extractivista y depredatoria que garantiza la expansión capitalista en el siglo XXI permea las prácticas y las maneras de sentir (y sentirse en) el mundo de los sujetos, pues, como sostiene Melucci (2016), el capital se expande mediante un concreto proceso de colonización del "planeta interno". En este marco, ¿qué tipos de individuos "fabrica” la segregación socio-espacial como fenómeno sistémico en las ciudades latinoamericanas?, ¿¿de qué formas cotidianas la segregación urbana profundiza y normaliza múltiples violencias asociadas con la distribución desigual de nutrientes, de derechos y de la palabra?, ¿qué modalidades de resistencias al orden imperante son posibles en el marco de ciudades cada vez más atravesadas por las desigualdades de género, raza y clase que se materializan en el espacio, organizando las experiencias de millones de sujetos?, ¿cuáles son las condiciones materiales y simbólicas para la singularización experiencial en las periferias pobres latinoamericanas?
} 
de los procesos y efectos de dominación que actualizan las percepciones asociadas con las formas socialmente construidas de las sensaciones. Así, en esta investigación se asume que experienciar la ciudad y los espacios que se habitan, lejos de remitir a un acto particular/individual, señala los modos socialmente construidos y aceptados de gestionar la distribución y disposición de clase de los cinco sentidos que organizan la vida social en general, y la vida urbana en particular (Cervio, 2015b).

El abordaje diacrónico de este tipo de experiencias requiere observar, junto con los condicionantes estructurales anteriormente mencionados, las distintas estrategias elaboradas individual y colectivamente por los sujetos para apropiarse del espacio, es decir, para convertir el espacio vivido en un lugar: esto es, adaptarlo, usarlo y transformarlo vertiendo en él afectos, creatividad y emociones (Lefebvre, 1972a, 1978a, 1978b). De allí la importancia cardinal que reviste para este análisis la tensión dialéctica entre "pruebas" y "soportes", como operadores analíticos que posibilitan interpretar las maneras en que lo social se estructura a nivel de las trayectorias y experiencias individuales.

Mientras las primeras constituyen los desafíos estructurales a través de los cuales los individuos "son producidos y se producen", los segundos son definidos como los medios a partir de los cuales el individuo llega a "sostenerse" en el mundo (Martuccelli, 2007). Para operacionalizar estos conceptos, en el marco de la investigación en curso, las pruebas remiten a los desafíos estructurales con los que los sujetos han tenido que batallar a lo largo de su vida y que, en su conjunto, definen los hitos de habitabilidad que éstos identifican en sus calendarios personales. Por su parte, los soportes son los elementos materiales, vinculares-afectivos y sensibles que caracterizan la vida cotidiana en los territorios, en los barrios y en los hogares, y que en su integralidad configuran las experiencias del habitar de los sujetos en clave biográfica.

Ahora bien, ¿por qué estudiar las conexiones entre segregación socioespacial, biografías y experiencias desde una lectura que privilegia el análisis de las sensibilidades? Frente a un proceso de segregación que se despliega iterativa e inter-generacionalmente en la periferia pobre platense, la opción por las trayectorias, retomando los aportes del enfoque biográfico, aparece como un camino analítico válido y metodológicamente potente. Como se afirmó, examinar los fenómenos sociales a escala del individuo no sólo posibilita observar la estructura, sino también la singularidad frente a la estandarización estructural. Es decir, permite indagar a) las maneras en que las vidas individuales son atravesadas y condicionadas por procesos estructurales y $b$ ) los modos como los sujetos enfrentan dichos desafíos, elaborando respuestas diferenciales y movilizando distintos tipos de recursos. 
Esta opción analítica sugiere un conjunto de desafíos teóricos, metodológicos y epistémicos que, a modo de cierre de este escrito, pueden sintetizarse del siguiente modo:

- Producir conocimiento científico sobre las conexiones entre procesos de estructuración socio-espaciales, biografías, experiencias y sensibilidades implica afianzar y refinar un enfoque teórico-epistémico que, al no aceptar ninguna clase de sustancialismo, está obligado a mostrar en forma permanente las conexiones entre evidencia empírica, herramientas teóricas e interpretaciones sociológicas. Tal posicionamiento demanda ejercer una continua vigilancia epistemológica atenta a la sistematicidad de las observaciones.

- Para comprender las trayectorias de habitabilidad, cuidando ajustar en forma rigurosa y metódica la aproximación (siempre externa/ extraña/extranjera) del analista al conocimiento experiencial que aportan los sujetos, es necesario desarrollar un acercamiento metodológico ad hoc, consistente con los objetivos de investigación y, al mismo tiempo, extremadamente cuidadoso de las historias personales y de las sensibilidades puestas en juego en cada situación de entrevista. Por ejemplo, Herbert Blumer (1982) sostenía que, si se parte del supuesto de que lo central de la vida social son los procesos de interpretación que realizan los sujetos, entonces las y los investigadores deben recurrir a "conceptos sensibilizadores" como guías de referencia para el planteo de las preguntas, la búsqueda de información y la interpretación de los datos. Es decir, se trata de definir conceptos abiertos para captar cómo se manifiestan y cuáles son las significaciones y sentidos que los sujetos atribuyen a cada palabra, a cada conflicto, a cada situación.

- Un abordaje como el propuesto requiere extremar las cautelas epistémicas ejerciendo una "actitud de escucha" activa. Tal actitud es comprendida aquí como un elemento cardinal para construir y sostener la relación que el analista establece a lo largo del trabajo de campo con los sujetos que participan en la investigación.

- Optar por el método biográfico para abordar las experiencias y sensibilidades de sujetos que (por generaciones) han hecho del habitar en la socio-segregación su manera de estar/sentir la ciudad, no significa la puesta en juego de modelos heurísticos deterministas y mecanicistas que deducen consecuencias micro-sociológicas de factores estructurales. Tampoco supone la opción por un individualismo metodológico que hace del individuo aislado su elemento de base. Por el contrario, los relatos biográficos posibilitan elaborar interpretaciones sobre las experiencias del habitar de los sujetos en 
un periodo de mediana duración, enfatizando una lectura sociológica de los fenómenos sociales por y desde las experiencias individuales. En otros términos, se trata de observar diacrónicamente los modos en que la experiencia individual se entreteje con la realidad social.

- La construcción colectiva del conocimiento que se produce mediante la utilización del método biográfico obliga a extremar la atención sobre la violencia epistémica que pueda infringirse durante el proceso. En particular, es preciso resguardar la intimidad de los sujetos que, al consentir ser partícipes de la investigación, están "relatando sus propias vidas" con fines académicos. En otros términos, elaborar un relato biográfico es compartir/desnudar frente al investigador/investigadora un conjunto de acontecimientos vividos, sobre los que se depositan ciertas valoraciones, emociones y conflictos. En algunos casos, también puede ser percibido como una posibilidad de "escucha" que los sujetos no encuentran o no tienen en otros espacios familiares o institucionales. Por ello, una escucha activa y respetuosa se impone como una demanda, la cual, además de metodológica, es en sí misma una actitud política y humana insoslayable de toda investigación comprometida con procesos de autonomía y emancipación social.

Financiamiento: Los avances y resultados discutidos en este escrito derivan de una investigación individual financiada y avalada por el Consejo Nacional de Investigaciones Científicas y Técnicas (Conicet), Argentina.

\section{Fuentes consultadas}

Araujo, Kathya y Martuccelli, Danilo (2012), Desafíos comunes. Retrato de la sociedad chilena y sus individuos, Santiago de Chile, LOM Ediciones.

Bericat Alastuey, Eduardo (1994), Sociología de la movilidad especial. El sedentarismo nómada, Madrid, Ediciones del Centro de Investigaciones Sociológicas.

Blumer, Herbert (1982), El interaccionismo simbólico. Perspectiva y Método, Barcelona, Hora. 
Borsdorf, Axel e Hidalgo Dattwyler, Rodrigo (2010), "From polarization to fragmentation. Recent changes in Latin American urbanization”, en Paul Van Lindert and Otto Verkoren (eds.), Decentralized development in Latin America: experiences in local governance and local development, Dordrecht, Springer, pp. 23-34, DOI 10.1007/97890-481-3739-8_2

Bourdieu, Pierre (1990), "Algunas propiedades de los campos", en Pierre Bourdieu, Sociología y cultura, Ciudad de México, Conaculta, pp.135-141.

Cervio, Ana (2019) "Desconfianza e interacciones urbanas. Un abordaje desde las sensibilidades sociales", en Ana Cervio y Brenda Bustos García (comps.), Confianza y politicas de las sensibilidades, Buenos Aires, Estudios Sociológicos.

Cervio, Ana (2015a), "Expansión urbana y segregación socio-espacial en la ciudad de Córdoba (Argentina) durante los años '80”, Astrolabio Nueva Época, núm. 14, Buenos Aires, Centro de Investigaciones y Estudios sobre Cultura y Sociedad (CIECS), Consejo Nacional de Investigaciones Científicas y Técnicas (Conicet) y Universidad Nacional de Córdoba (UNC), pp. 360-392.

Cervio, Ana. (2015b) "Experiencias en la ciudad y políticas de los sentidos. Lecturas sobre la vista, el oído y el olfato”, en Rafael Sánchez Aguirre (comp.), Sentidos y sensibilidades: exploraciones sociológicas sobre cuerpos-emociones, Buenos Aires, Estudios Sociológicos Editora, pp. 17-48.

Cervio, Ana y Vergara, Gabriela (2017), “Segregación socio-espacial, conflictos y sensibilidades: disputas por la movilidad y el desplazamiento en la ciudad de Córdoba, Argentina", Aposta. Revista de Ciencias Sociales, núm. 74, Madrid, Apostadigital.com, pp. 111-144.

Corominas, Joan y Pascual, José Antonio (1984), Diccionario crítico etimológico castellano e hispánico, vol. III, Madrid, Gredos.

Delaunay, Daniel y Dureau, Françoise (2004), “Componentes sociales y espaciales de la movilidad residencial en Bogotá”, Estudios demográficos y urbanos, 19 (1), Ciudad de México, Centro de Estudios Demográficos, Urbanos y Ambientales de El Colegio de México, pp. 77-113. 
Di Virgilio, Mercedes (2009), “Trayectorias residenciales y estrategias habitaciones entre familias de sectores populares y medios residentes en el Área Metropolitana de Buenos Aires, Argentina”, en Jaime Fabián Erazo Espinosa (coord.), Inter/secciones urbanas: origen y contexto en América Latina, Quito, Facultad Latinoamericana de Ciencias Sociales (FLACSO Ecuador) y Ministerio de Cultura del Ecuador, pp. 233-257.

Duhau, Emilio (2003), "División social del espacio metropolitano y movilidad residencial”, Papeles de Población, 9 (36), Toluca, Centro de Investigación y Estudios Avanzados de la Población, Universidad Autónoma del Estado de México, pp. 161-210.

Frediani, Julieta Constanza (2013), "La problemática del hábitat informal en áreas periurbanas del partido de La Plata", Revista Universitaria de Geografía, 22 (1-2), Bahía Blanca, Departamento de Geografía y Turismo, Universidad Nacional del Sur, pp. 43-67.

Frediani, Julieta (2010), "Lógicas y tendencias de la expansión residencial en áreas periurbanas. El Partido de La Plata, Buenos Aires, Argentina, entre 1990 y 2010", tesis doctoral, Universidad Nacional de La Plata, La Plata.

Frediani, Julieta; Rodríguez Tarducci, Rocío y Cortizo, Daniela (2018), "Proceso de Gentrificación en Áreas Periféricas del Partido de La Plata, Argentina”, Quid 16, núm. 9, Buenos Aires, Instituto de Investigaciones Gino Germani, pp. 9-3.

Gobierno de la Provincia de Buenos Aires (2015), "Registro Público de Villas y Asentamientos de la Provincia de Buenos Aires", Buenos Aires, Ministerio de Infraestructura y Servicios Públicos, Gobierno de la Provincia de Buenos Aires, <http://www.mosp.gba.gov.ar/ subsecretarias/reg_vilyasent.php>, 4 de marzo de 2019.

Heidegger, Martin (1951), Construir, habitar, pensar. Alemania, Darmstadt, <http://www.fadu.edu.uy/estetica-diseno-ii/files/2013/05/ Heidegger-Construir-Habitar-Pensar1.pdf>, 15 de junio de 2018.

INDEC (Instituto Nacional de Estadísticas y Censos) (2012), “Censo Nacional de Población, Hogares y Viviendas 2010. Censo del Bicentenario. Resultados definitivos", Buenos Aires, INDEC. 
Le Breton, David (2009), El sabor del mundo. Una antropología de los sentidos, Buenos Aires, Nueva Visión.

Lefebvre, Henri (2013), La producción del espacio, Madrid, Capitán Swing. Lefebvre, Henri (1978a), De lo rural a lo urbano, Barcelona, Península.

Lefebvre, Henri (1978b), El derecho a la ciudad, Barcelona, Península.

Lefebvre, Henri (1972a), La revolución urbana, Madrid, Alianza.

Lefebvre, Henri (1972b), La vida cotidiana en el mundo moderno, Madrid, Alianza.

Lévy, Jean Pierre y Brun, Jacques (2002), "Introducción. De la extensión a la renovación metropolitana: mosaico social y movilidad", en Françoise Dureau; Véronique Dupont; Éva Lelièvre; Jean-Pierre Lévy; Thierry Lulle (coords.), Metrópolis en movimiento: Una comparación internacional, Bogotá, Instituto de Investigaciones para el Desarrollo, pp. 147-161.

Lindon Villoria, Alicia (2009), "La construcción socioespacial de la ciudad: el sujeto cuerpo y el sujeto sentimiento", Revista Latinoamericana de Estudios sobre Cuerpos, Emociones y Sociedad, núm. 1, Buenos Aires, Centro de Investigaciones y Estudios Sociológicos, pp. 6-20.

Martuccelli, Danilo (2007), Cambio de rumbo. La sociedad a escala del individuo, Santiago de Chile, LOM Ediciones.

Martuccelli, Danilo y Singly, François de (2012), Las sociologías del individuo, Santiago de Chile, LOM Ediciones.

Marx, Karl (2010) Manuscritos de 1844. Economía politica y filosofía, Buenos Aires, Colihue.

Melucci, Alberto (2016) Cuerpos extraños, Buenos Aires, Estudios Sociológicos Editora.

Mongin, Olivier (2006), La condición urbana. La ciudad a la hora de la mundialización, Buenos Aires, Paidós.

Prévôt Schapira, Marie France (2002), "Fragmentación espacial y social: conceptos y realidades”, Perfiles Latinoamericanos, 9 (19), Ciudad 
de México, Facultad Latinoamericana de Ciencias Sociales (FLACSO México), pp. 33-56.

Prèvôt-Schapira, Marie France (2000), “Segregación, fragmentación, secesión. Hacia una nueva geografía social en la aglomeración de Buenos Aires", Revista Economía, Sociedady Territorio, 2 (7), Toluca, El Colegio Mexiquense A. C., pp. 405-431, DOI: http://dx.doi. org/10.22136/est002000437

Pujadas Muñoz, Juan José (1992), El método biográfico: El uso de las historias de vida en ciencias sociales, Madrid, Centro de Investigaciones Sociológicas.

RAE (Real Academia Española (2019), Diccionario de la Lengua Española, Madrid, Espasa, <https://dle.rae.es/>, 5 de marzo de 2018.

Rebord, Gustavo (2006), "La política oficial hacia los asentamientos irregulares en la ciudad de Córdoba en el período 1970-2006. El Programa 'Mi casa Mi vida", ponencia presentada en el Seminario Latinoamericano "Teoría y Política sobre asentamientos informales", 8 y 9 de noviembre, Buenos Aires, Universidad Nacional de General Sarmiento.

Río, Juan Pablo del (2016), “Tensiones entre hipoteca, suelo y política urbana. El caso Pro.Cre.Ar en el Partido de La Plata, provincia de Buenos Aires", Estudios Socioterritoriales. Revista de Geografía, núm. 19, Tandil, Centro de Investigaciones Geográficas, Universidad Nacional del Centro de la Provincia de Buenos Aires (UNCPBA) y Consejo Nacional de Investigaciones Científicas y Técnicas (Conicet), pp. 135-151.

Rodríguez Tarducci, Rocío (2018), “Asentamientos informales en el Partido de La Plata. Una aproximación a las modalidades de ocupación del territorio", Estudios Socioterritoriales. Revista de Geografía, núm. 23, Tandil, Centro de Investigaciones Geográficas, Universidad Nacional del Centro de la Provincia de Buenos Aires (UNCPBA) y Consejo Nacional de Investigaciones Científicas y Técnicas (Conicet), pp. 119-136.

Sabatini, Francisco; Cáceres, Gonzalo y Cerda, Jorge (2001), "Segregación residencial en las principales ciudades chilenas: Tendencias de las tres últimas décadas y posibles cursos de acción”, Revista Latinoamericana de Estudios Urbano Regionales, 27 (82), Santiago de 
Chile, EURE, <http://www.eure.cl/index.php/eure/article/ view/1258>, 25 de junio de 2018.

Saraví, Gonzalo (2008), "Mundos aislados: segregación urbana y desigualdad en la ciudad de México”, Revista EURE, 34 (103), Santiago de Chile, EURE, pp. 93-110.

Sautu, Ruth (1999), "Estilos y prácticas de la investigación biográfica”, en Ruth Sautu (comp.), El método biográfico. La reconstrucción de la sociedad a partir del testimonio de los actores, Buenos Aires, Editorial Belgrano, pp. 21-59.

Sennet, Richard (2007), Carne y Piedra. El cuerpo y la ciudad en la civilización occidental, Madrid, Alianza.

Scribano, Adrián (2017), "Amor y acción colectiva: una mirada desde las prácticas intersticiales en la Argentina”, Aposta. Revista de Ciencias Sociales, núm. 74, Madrid, Apostadigital.com, pp. 241-280.

Scribano, Adrián (comp.) (2013), Teoría social, cuerpos y emociones, Buenos Aires, Estudios Sociológicos Editora.

Scribano, Adrián (2010), "Primero hay que saber sufrir...!!! Hacia una sociología de la 'espera' como mecanismo de soportabilidad social”, en Adrián Scribano y Pedro Lisdero (comps.), Sensibilidades en juego: miradas múltiples desde los estudios sociales de los cuerpos y las emociones, Córdoba, Centro de Estudios Avanzados-Consejo Nacional de Investigaciones Científicas y Técnicas, pp. 169-192.

TECHO (2016), "Relevamiento de asentamientos informales 2016", Buenos Aires, TECHO, <http://relevamiento.techo.org.ar/downloads/informe_relevamiento_TECHO_2016.pdf>, 4 de septiembre de 2018.

Varela, Omar y Cravino, María (2008), “Mil nombres para mil barrios. Los asentamientos y villas como categorías de análisis y de intervención", en María Cravino (coord.) Los mil barrios (in)formales Aportes para la construcción de un observatorio del hábitat popular del Área Metropolitana de Buenos Aires, Los Polvorines, Universidad Nacional de General Sarmiento, pp. 45-64.

Vecslir, Lorena y Ciccolella, Pablo (2012),’Transformaciones territoriales recientes y reestructuración metropolitana en Buenos Aires”, 
Revista Iberoamericana de Urbanismo (RIURB), núm. 8, Barcelona, RIURB, pp. 1-7, <https://upcommons.upc.edu/handle/ 2099/13011>, 25 de octubre de 2019.

Wright Mills, Charles (2003), La imaginación sociológica, Ciudad de México, Fondo de Cultura Económica.

Recibido: 5 de septiembre de 2019. Reenviado: 8 de noviembre de 2019. Aceptado: 17 de enero de 2020.

Ana Lucía Cervio. Doctora en Ciencias Sociales por la Universidad de Buenos Aires, Argentina. Actualmente, es investigadora asistente del Consejo Nacional de Investigaciones Científicas y Técnicas (Conicet) con lugar de trabajo en el Centro de Investigaciones sobre Comunidad Local, Participación y Política Social (CICLOP), Universidad de Buenos Aires (UBA), investigadora del Centro de Investigaciones y Estudios Sociológicos (CIES), editora y coordinadora general de la Revista Latinoamericana de Metodología de la Investigación Social(ReLMIS). Sus líneas de investigación actuales son las experiencias, conflictos y sensibilidades urbanas, desde una sociología de los cuerpos y las emociones. Entre sus últimas publicaciones destacan: "Política alimentaria, pobreza y emociones en la Argentina de los años 80", Revista Entramado, 15 (1), Cali, Universidad Libre, pp. 62-77 (2019); “¿Qué te pasa Buenos Aires? La inseguridad como una "práctica del sentir" porteño", Revista Brasileira de Sociologia da Emoção, 18 (52), Paraíba, Grupo de Pesquisa em Antropologia e Sociologia das Emoçóes (GREM)-Universidade Federal da Paraíba, pp. 75-90 (2019); en coautoría: Sensibilidades y experiencias: acentos, miradas y recorridos desde los estudios sociales de los cuerpos/emociones, Buenos Aires, Estudios Sociológicos Editora (2018); en coautoría, “Distrust and Proximity. The Paradoxes of Violence in Argentina”, en Adrián Scribano, Politics and Emotions, Houston, Studium Press, pp. 193-219 (2018). 